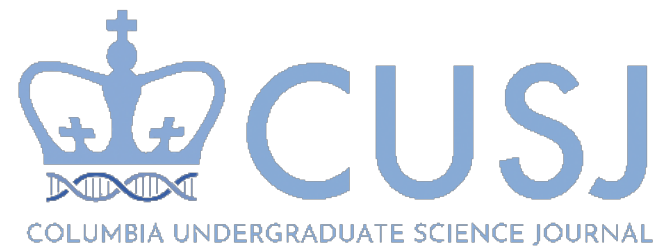

\title{
Effect of Eccentricity in Microwave Imaging of Multiple Composite Pipes
}

\author{
Yuki Gao*1, Noshin Raisa1, and Reza K. Amineh \\ ${ }^{1}$ Department of Electrical and Computer Engineering, New York Institute of Technology, New York, \\ NY, USA \\ KEYWORDS: Eccentricity effect, microwave imaging, non-destructive testing, non-metallic pipes
}

ABSTRACT: The use of non-metallic composites that are durable, low cost, and lightweight is growing fast in various industries. In the oil and gas industry, a commonly used form of these materials is in the shape of pipes. Such pipes can be damaged due to material loss (defects and holes), erosions, and more which may cause major production failures or environmental mishaps. To prevent these issues, non-destructive testing (NDT) methods need to be employed for regular inspections of such components. Since traditional NDT methods are mainly used for metallic pipes, microwave imaging has recently been proposed as a promising approach for examination of non-metallic pipes. While microwave imaging can be employed for inspection of multiple layers of pipes, the effect of undesired eccentricity of the pipes (undesired distance between the centers of multiple pipes which are supposed to be concentric) can impose additional imaging errors. In this paper, for the first time, we study the effect of eccentricity of the pipes on the images reconstructed using near-field holographic microwave imaging on double pipes through simulations. To have a realistic study, we add artificial noise to the simulated data.

\section{INTRODUCTION}

Recently, non-metallic pipes and composite components such as fiber reinforced plastic (FRP), glass reinforced epoxy resin (GRE), high density polyethylene (HDPE), reinforced rubber expansion joints (REJs), carbon fiber reinforced plastics (CFRP), and polyvinyl chloride (PVC) are replacing metallic pipes throughout different industries due to advantages such as durability, low cost, light-weight, resistance to corrosion, etc. The growing demand for these materials necessitates the use of proper non-destructive testing (NDT) techniques for material integrity inspections. In general, NDT methods such as ul- trasonic testing, radiography, eddy current, and magnetic flux leakage have been widely applied in different industries for inspection of metallic components. However, these NDT methods cannot fulfill the demand for testing certain materials and components such as non-metallic composite pipes. For example, due to the complex structure of composite materials such as FRP/GRE [1] and the nature of defects and failure morphology in HDPE thermal fusion joints, ultrasonic testing fails to perform NDT for these mediums [2, 3]. On the other hand, radiography relies on the use of X-ray [4] which requires extra safety measures. Besides, it is incapable of detecting de-

(C) 2021 Gao, Raisa, Amineh. This is an open access article distributed under the terms of the Creative Commons Attribution License, which permits the user to copy, distribute, and transmit the work provided that the original authors and source are credited. 
lamination and planar cracks for defects when the local density remains nearly the same.

Thus, to fulfill the growing demand for NDT of non-metallic materials, microwave measurement techniques have been proposed (e.g., see [5, 6]). The usage of microwave imaging helps detecting defects, cracks, holes, and more in such components. In particular, microwave holographic imaging is a fast and robust imaging technique that has been successfully applied in various applications such as the security screening of airport passengers [7], etc. Originally, microwave holographic imaging techniques were developed based on synthetic aperture radar (SAR) imaging techniques [7] which employ far-field assumptions, i.e., the imaging distance which is the distance between the measuring antennas and the imaged object is assumed to be much larger than the wavelength. Wide-band SAR imaging has been used to produce three-dimensional (3D) images of the vertical cracks/flaws in fat and curved HDPE pipes [8]. Recently, SAR-based imaging techniques have been extended to the near-field applications where the distance between the measuring antennas and the imaged object is small (e.g., see [9, 10]). Thus, these techniques can be called near-field holographic imaging techniques where the information related to a specific imaging system is obtained a priori through the measurement of the so-called point-spread functions (PSFs) [11]. This offers several advantages such as: the reduction of modeling errors (as the modeling of the antennas and the imaging setup is not required), the reduction of errors due to uncertainties in the material properties, and the reduction of errors due to the size of antennas (measuring the PSFs directly instead of having assumption-based point-wise antennas). Although analytical expressions for the PSF (instead of direct measurement of them) can still be used in near-field holographic imaging, the material selection and the ignored near-field terms for the antennas may degrade the image reconstruction quality.

It is common to use multiple pipes in concentric configuration, as illustrated in Figure 1 , to improve the efficiency and increase the lifetime of the wellbore production in oil and gas industry [12] or to separate the flow in the fluid transfer pipeline [13]. Near-field holographic imaging has been extended to the application of multiple non-metallic pipe imaging in [14, 15] where the pipes are assumed to be perfectly concentric. In this paper, we study the performance of the near-field holographic imaging of double pipes with different eccentricity values, i.e., the centers of the two pipes are not perfectly aligned. Although, in industry, normally centralizers are employed for making the multiple pipes concentric, the small misalignment of the centers, called eccentricity, can impose errors in image reconstruction when using techniques that have been developed based on the zero-eccentricity assumption. Thus, here, we consider this important factor for the first time and we use a quantitative measure, called reconstruction error (RE), to evaluate the degradation of the images of the defects on the inner and outer pipes of a double-pipe configuration due to various eccentricity values. It is worth noting that the effect of other important parameters for the considered microwave imaging setup such as thickness, radius, and permittivity of the pipes as well as angular separation of the antennas have been already studied in [15] and will be excluded here. Although the study is performed through simulations, we add artificial noise to the simulated data to have realistic results.

\section{METHODS}

In this section, we review the near-field holographic imaging approach for imaging of multiple pipes using an array of receiver antennas and multiple frequency data. Figure 1 illustrates the microwave imaging setup. It consists of a transmitter antenna to illuminate the pipes and an array of $N_{A}$ receiver antennas measuring the scattered fields. The transmitter and receiver antennas scan a circular aperture with radius of $r_{A}$. It is assumed that the defects and 
pipes are infinite along the longitudinal direction ( $z$ ). The scattered field is recorded at $N_{\phi}$ angles along the azimuthal direction $\phi$ (within $[0,2 \pi])$. The complex-valued scattered field $E^{S C}(\phi)$ is measured, at each sampling position, at $N_{\omega}$ frequencies within the band of $\omega_{1}$ to $\omega_{N_{\omega}}$ by each receiver. Such scattered response is obtained from subtracting the response of the pipes without defects from the response of the same pipes with defects. The image reconstruction process then provides one-dimensional (1D) images of the pipes with radii $r_{i}$, where $i=1, \ldots, N_{r}$. Please note that the imaging along the $z$ direction can be implemented using similar concepts discussed here. The imaging system is assumed to be linear and space-invariant (LSI) which allows us to use the convolution theory. The convolution theory allows to write the response to an unknown input to the system as the convolution of the point-spread functions (PSF) (also known as impulse responses) of the system with that unknown input function.

For implementation of the near-field holographic imaging, first, the PSFs of the LSI imaging system are acquired. These PSFs are approximated by measuring small defects, called calibration defects (CDs) placed on each pipe one at a time, representing impulse functions as the inputs to the imaging system. In other words, the PSFs are measured by the same imaging system that will be later used for imaging test objects. These CDs are the smallest defects that can be measured by the system. To provide more data for image reconstruction, measurements can be implemented at multiple frequencies, $\omega_{n}, n=1, \ldots, \quad N_{\omega}$ and by multiple receivers, $a_{m}, m=1, \ldots, N_{A}$. We denote the measured PSF function for the $i$-th pipe measured by the receiver antenna $a_{m}$ at frequency $\omega_{n}$ by $E_{i, a_{m}}^{S C, C D}\left(\phi, \omega_{n}\right)$. We also denote the measured scattered field by the receiver antenna $\mathrm{a}_{\mathrm{m}}$ at frequency $\omega_{n}$ by $E_{a_{m}}^{S C}\left(\phi, \omega_{n}\right)$. Let's first consider the spatially-sampled versions of $E_{a_{m}}^{S C}\left(\phi, \omega_{n}\right), E_{i, a_{m_{B}}}^{S C, C D}\left(\phi, \omega_{n}\right)$, and $f_{i}(\phi)$ denoted by $\mathbf{E}_{a_{m}}^{S C C}\left(n_{\phi}, \omega_{n}\right), \mathbf{E}_{i, a_{m}}^{S C, C D}\left(n_{\phi}, \omega_{n}\right)$, and $\mathbf{f}_{i}\left(n_{\phi}\right), n_{\phi}=1, \ldots, N_{\phi}$ , with the angular interval denoted by $\Delta \phi$. Us-

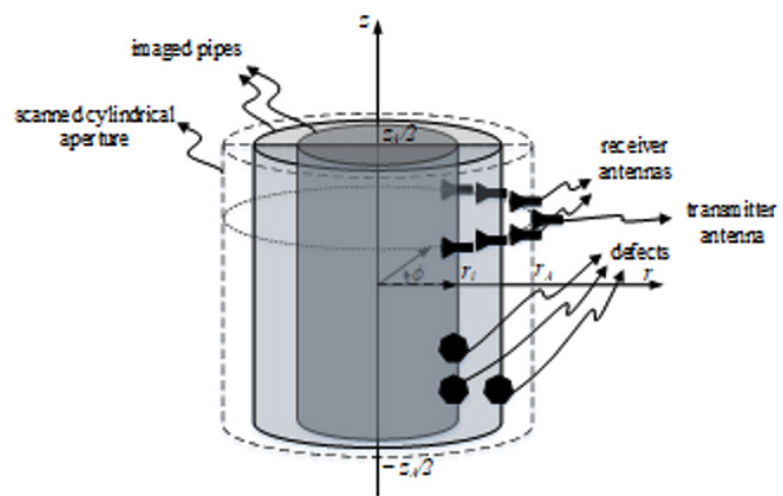

Figure 1: Illustration of the simulation setup in FEKO for the case that the defects are on the inner pipe.

ing the convolution theory and discrete Fourier transforms (DFT) along $\phi$ direction, it can be shown that the unknown shape functions of the defects on the pipes $f_{i}(\phi), i=1, \ldots, N_{r}$, can be found by solving the following system of equations at each spatial frequency $k_{\phi}$ [15]:

$$
\underline{\tilde{\mathbf{E}}}^{S C}=\underline{\tilde{\mathbf{D}}} \tilde{\tilde{\mathbf{F}}}
$$

where

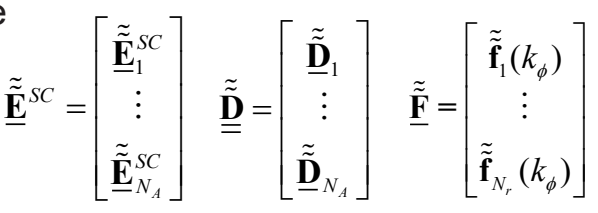

and

$$
\begin{array}{r}
\tilde{\tilde{\mathbf{E}}}_{a_{m}}^{S C}=\left[\begin{array}{c}
\tilde{\tilde{\mathbf{E}}}_{a_{m}}^{S C}\left(k_{\phi}, \omega_{1}\right) \\
\vdots \\
\tilde{\tilde{\mathbf{E}}}_{a_{m}}^{S C}\left(k_{\phi}, \omega_{N_{\omega}}\right)
\end{array}\right] \\
\tilde{\tilde{\mathbf{D}}}_{a_{m}}=\left[\begin{array}{ccc}
\tilde{\tilde{\mathbf{E}}}_{1, a_{m}}^{S C, C D}\left(k_{\phi}, \omega_{1}\right) & \cdots & \tilde{\tilde{\mathbf{E}}}_{N_{r}, a_{m}}^{S C, C D}\left(k_{\phi}, \omega_{1}\right) \\
\vdots & \ddots & \vdots \\
\tilde{\tilde{\mathbf{E}}}_{1, a_{m}}^{S C, C D}\left(k_{\phi}, \omega_{N_{\omega}}\right) & \cdots & \tilde{\tilde{\mathbf{E}}}_{N_{r}, a_{m}}^{S C, C D}\left(k_{\phi}, \omega_{N_{\omega}}\right)
\end{array}\right]
\end{array}
$$

where $\quad \tilde{\tilde{\mathbf{E}}}_{a_{m}}^{S C}\left(k_{\phi}, \omega_{n}\right), \quad \tilde{\tilde{\mathbf{E}}}_{i, a_{m}}^{S C, C D}\left(k_{\phi}, \omega_{n}\right)$, and $\quad \tilde{\tilde{\mathbf{f}}}_{i}\left(k_{\phi}\right)$ denote DFT along $\phi$ axis of $\mathbf{E}_{a_{m}}^{S C}\left(n_{\phi}, \omega_{n}\right)$, $\mathbf{E}_{i, a_{m}}^{S C, C D}\left(n_{\phi}, \omega_{n}\right)$, and $\mathbf{f}_{i}\left(n_{\phi}\right)$, respectively. These systems of equations are solved at each spatial frequency $k_{\phi}$ to obtain the values for $\tilde{\mathbf{f}}_{i}\left(k_{\phi}\right), \quad i=1, \ldots, N_{r}$. Then, inverse DFT along $\phi$ is applied to reconstruct images $\mathbf{f}_{i}\left(n_{\phi}\right)$ over all the pipes with radii $r=r_{i}, i=1, \ldots, N_{r}$. At the end, the normalized modulus of $\mathbf{f}_{i}\left(n_{\phi}\right)$ 
$\left|\mathbf{f}_{i}\left(n_{\phi}\right)\right| / M$, where $M$ is the maximum of $\left|\mathbf{f}_{i}\left(n_{\phi}\right)\right|$ for all $r_{i}$, is plotted versus $\phi$ to obtain a 1D image of the defects on the i-th pipe. We call $\left|\mathbf{f}_{i}\left(n_{\phi}\right)\right| / M$ the normalized image.

In [15], inspired by standardized low-resolution brain electromagnetic tomography, the systems of equations in (1) are solved using standardization of the minimum norm. Using this concept, the objective function to be minimized is constructed as:

$$
J=\left\|\underline{\tilde{\mathbf{E}}}^{S C}-\underline{\tilde{\mathbf{D}}} \tilde{\tilde{\mathbf{F}}}\right\|^{2}+\alpha\|\tilde{\tilde{\mathbf{F}}}\|
$$

where $\alpha \geq 0$ is a regularization parameter. The detailed solution has been explained in [15].

As discussed earlier, the PSF data is collected beforehand for the configuration of the pipes under inspection assuming that they are concentric. A non-zero eccentricity, however, affects the measured data for the inspected pipes leading to errors in the reconstructed images. To evaluate the quality of reconstructed images, we define a reconstruction error (RE) parameter as:

$$
\mathrm{RE}=\sum_{i=1}^{N_{r}}\left\|\mathbf{f}_{i}\left(n_{\phi}\right) \mid / M-\mathbf{f}_{i, \text { ideal }}\left(n_{\phi}\right)\right\|
$$

where $\mathbf{f}_{i, \text { ideal }}\left(n_{\phi}\right)$ is the ideal image for which the values are all 0 except being 1 at the true positions of the defects.

\section{RESULTS}

To study the effect of eccentricity on imaging of the multiple non-metallic composite pipes, we conduct a study using simulation data provided by Altair's FEKO software [16] which is a high frequency modeling software. The study was done by $1 \mathrm{D}$ scanning and image reconstructions along the azimuthal direction. In order to have a more realistic simulation study, white Gaussian noise with signal-to-noise ratio (SNR) of $20 \mathrm{~dB}$ is added to the simulated responses by using the awgn command in MATLAB.

Figure 2 illustrates the configuration of the imaging setup in FEKO. We study the performance of the system where the antenna array is placed on the outside of two concentric pipes. There are 13 resonant dipole antennas separated by $\Delta \phi_{a}=10^{\circ}$ angles along the $\phi$ direction. Thus, all the antennas are used as receivers except the center element which acts as both transmitter and receiver. The radii of the inner and outer pipes, namely, $R_{\text {out } 1}$ and $R_{\text {out } 2}$ are $20 \mathrm{~mm}$ and $40 \mathrm{~mm}$, respectively, and the thickness of both pipes is $D=2 \mathrm{~mm}$. The pipes have a relative permittivity $\varepsilon_{r}$ of 2.25 and a tangent loss of 0.0004 . The defects have semi-cylindrical shape and their parameters are $L_{d}=1.5 D$ and $W_{d}=0.75 D$. In addition, the models are simulated with two identical defects on the pipes. The studied scenarios are: (1) both defects on the outer pipe only and (2) both defects on the inner pipe only. The eccentricity parameter, denoted by $E_{c c}$, represents the distance between the centers of the inner and outer pipes (the outer pipe is assumed to be concentric with the circular path scanned by the antennas known as measurement aperture).

For data acquisition, we perform scanning of a circular aperture to get the complex-valued transmission scattering parameters (in microwave, these are the parameters representing the coupling of the transmitter to

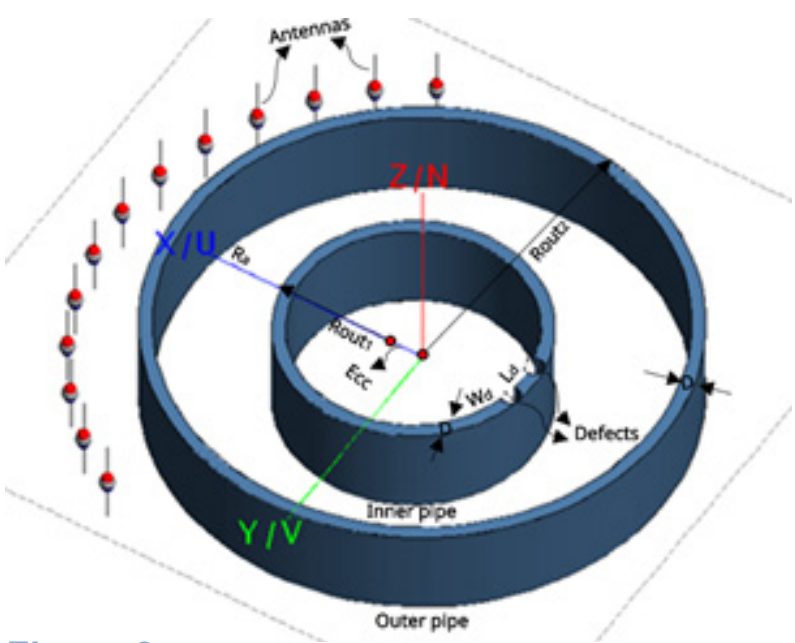

Figure 2: Illustration of the simulation setup in FEKO for the case that the defects are on the inner pipe. 
the receiver which here takes into account the field scattered back from the objects as well) by rotating the antennas along the azimuth angle $(\phi)$ from $0^{\circ}$ to $360^{\circ}$ every $2^{\circ}$ (181 grid points) in FEKO. For each scenario, the simulated responses without the presence of the defects are subtracted from the simulated responses with the presence of the defects to acquire the scattered responses only due to the defects. Also, white Gaussian noise with SNR of $20 \mathrm{~dB}$ is added to each defect response to imitate real-world measurement data.

First, we study the effect of eccentricity when $E_{c c}=0.5 \mathrm{~mm}$ (along the $x$ axis) and both defects are on the inner pipe or on the outer pipes at various azimuthal angles from $\phi= \pm 10^{\circ}$ to $\phi= \pm 170^{\circ}$.

After applying near-field holographic imaging as described in the previous section along with the PSF data collected for concentric pipes, the values of REs are computed for each scenario. Figures $3 \mathrm{~A}$ and $3 \mathrm{~B}$ show the variations of the computed REs versus the angle of defects for the cases that both defects are on the outer pipe and inner pipe, respectively. From both figures, it is observed that there is no clear correlation between the an- gles of the defects and the values of the REs. In general, the error seems to be larger for the defect angles between $\phi= \pm 20^{\circ}$ to $\phi= \pm 140^{\circ}$ - Furthermore, the values of REs are larger when the defects are on the inner pipe indicating that the image of the inner pipe is more affected by the adverse effects of eccentricity.

Next, we study the effect of value of eccentricity on the quality of the reconstructed images when the defects are on the outer and inner pipes by visually comparing the quality of the reconstructed images to the ideal images. For this study, the eccentricity parameter $E_{c c}$ varies from $0.1 \mathrm{~mm}$ to $0.9 \mathrm{~mm}$ with steps of $0.1 \mathrm{~mm}$ and we choose constant angles of $\phi= \pm 170^{\circ}$ for the defects one time when they are on the outer pipe and another time when the defects are on the inner pipes.

Figure 4 shows the reconstructed images of the two pipes when the eccentricity parameter is $0.1 \mathrm{~mm}$. Figures $4 \mathrm{~A}$ and $4 \mathrm{~B}$ show the images when the defects are on the outer and inner pipes respectively. In general, we notice that the image deteriorates is more for the inner pipe than the outer pipe. The reconstructed image in Figure 4A for which the defects are on the outer pipe clearly shows the

B

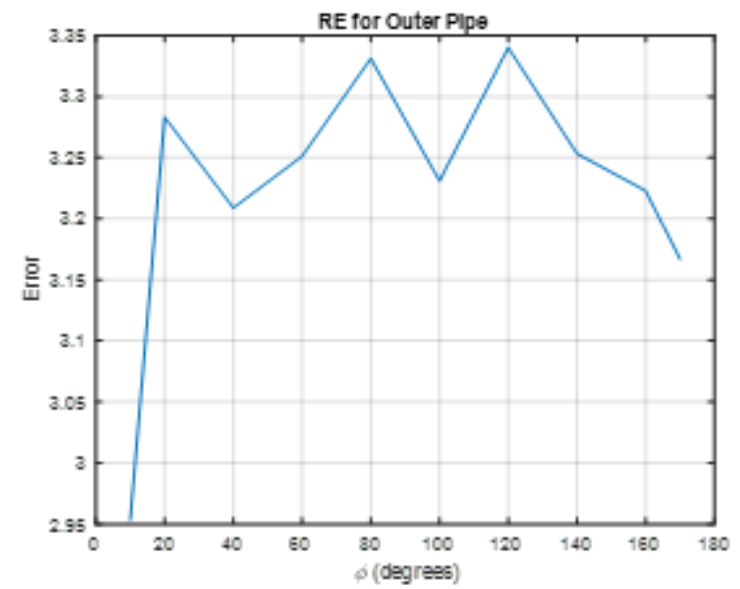

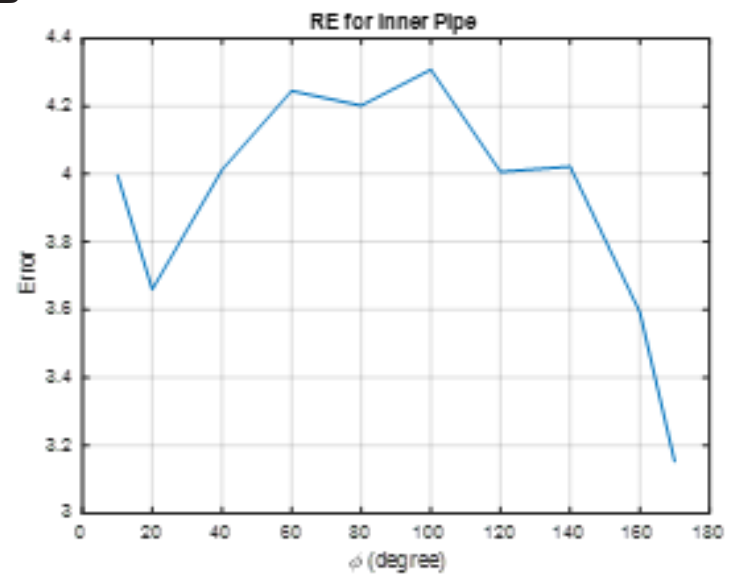

Figure 3: Variation of computed $\mathrm{RE}$ when $E_{c c}=0.5 \mathrm{~mm}$ and the angular positions of two identical defects are varying from $\phi= \pm 10^{\circ}$ to $\phi= \pm 170^{\circ}$ :

(A) defects are on outer pipe

(B) defects are on inner pipe. 
presence of the defects and it is close to the ideal image. However, the image for the inner pipe in Figure 4A shows artifacts around 0.3 level. The reconstructed images in Figure 4B in which the defects are on the inner pipe, still show the presence of the defects on the inner pipes but again contains large artifacts with maximum of 0.5 level. In this case, the

A

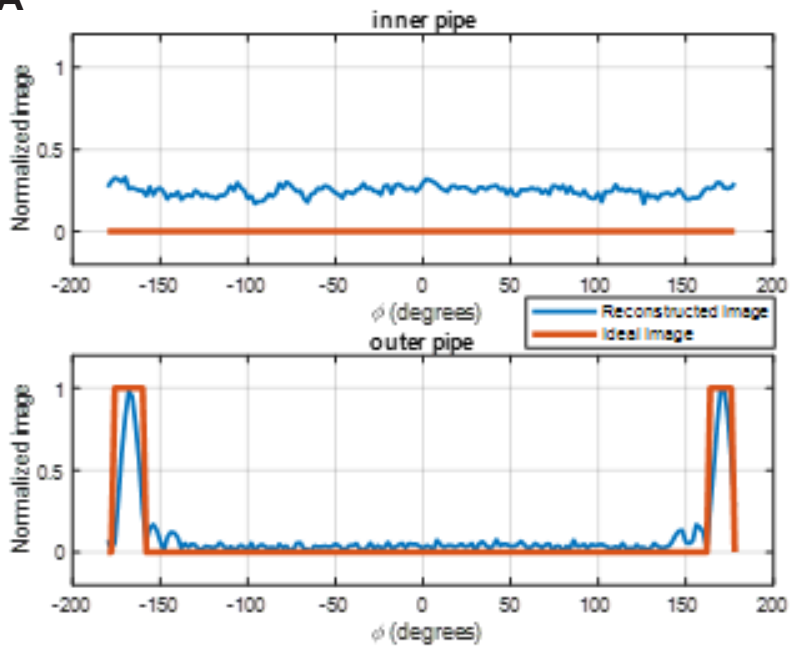

image on the outer pipe also contains some small level (around 0.1 level) of artifacts with some shadows of the defects on the inner pipe.

In the following, we demonstrate that as we continue to increase the value of eccentricity parameter in this study, the quality of the reconstruction images deteriorates significantly. Figure 5 shows the reconstructed images when

B

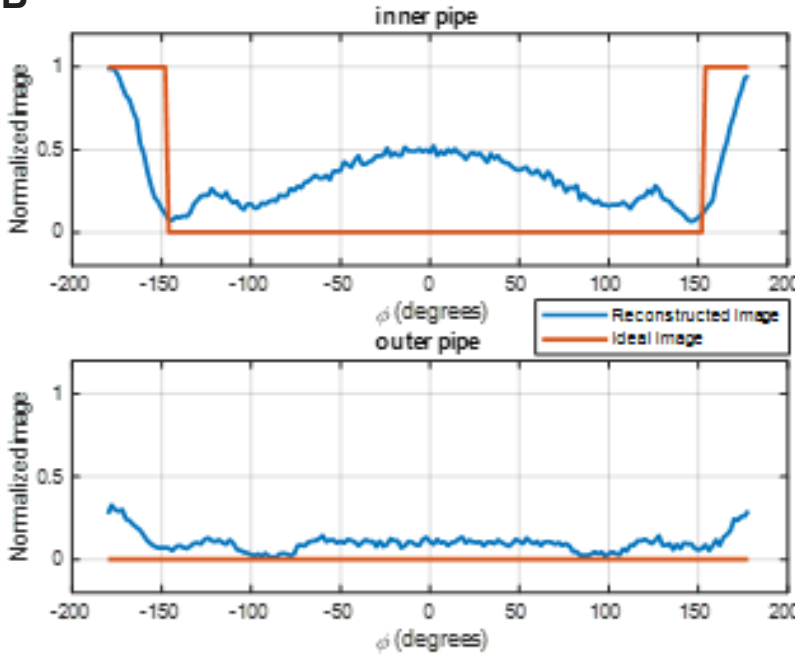

Figure 4: Reconstructed 1D images when the defects are at $\phi= \pm 170^{\circ}$ and eccentricity $=0.1 \mathrm{~mm}$ for:

(A) defects on the outer pipe

(B) defects on the inner pipe.

A
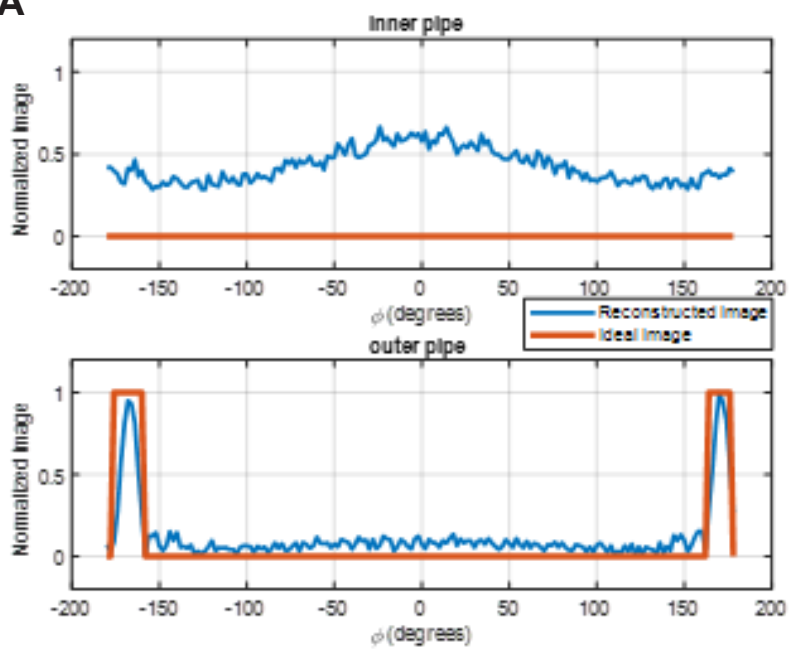

B
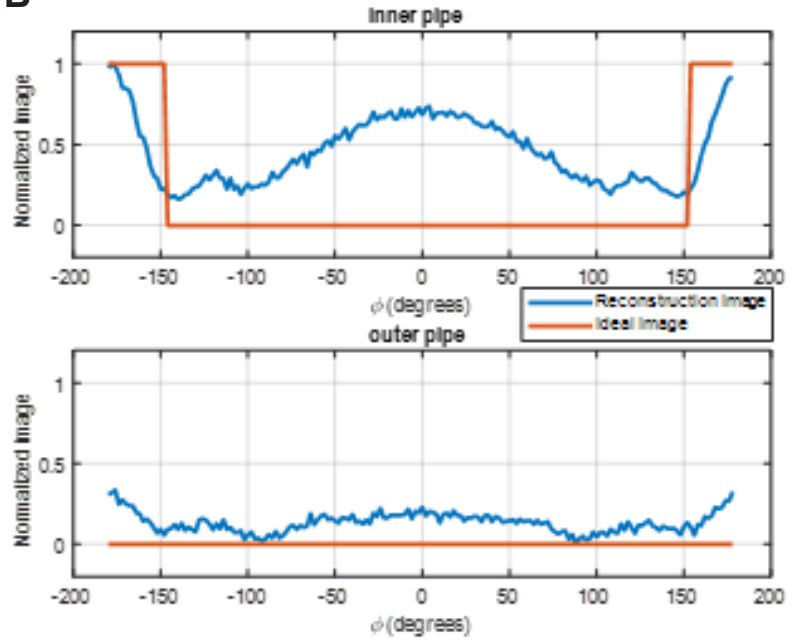

Figure 5: Reconstructed 1D images when the defects are at $\phi= \pm 170^{\circ}$ and eccentricity $=0.5 \mathrm{~mm}$ for:

(A) defects on the outer pipe

(B) defects on the inner pipe. 
the value of $E_{c c}$ is increased to $0.5 \mathrm{~mm}$. Compared to Figure 4, images in Figure 5 are more distorted. As expected, the increase in the value of $E_{c c}$ leads to larger image reconstruction errors. Next, we increase the value of $E_{c c}$ even further. Figure 6 displays the high deterioration of the images when the value of $E_{c c}$ is $0.9 \mathrm{~mm}$. From these figures, it can be easily deduced that the reconstructed image is far off from the ideal image due to high error caused by eccentricity.

A
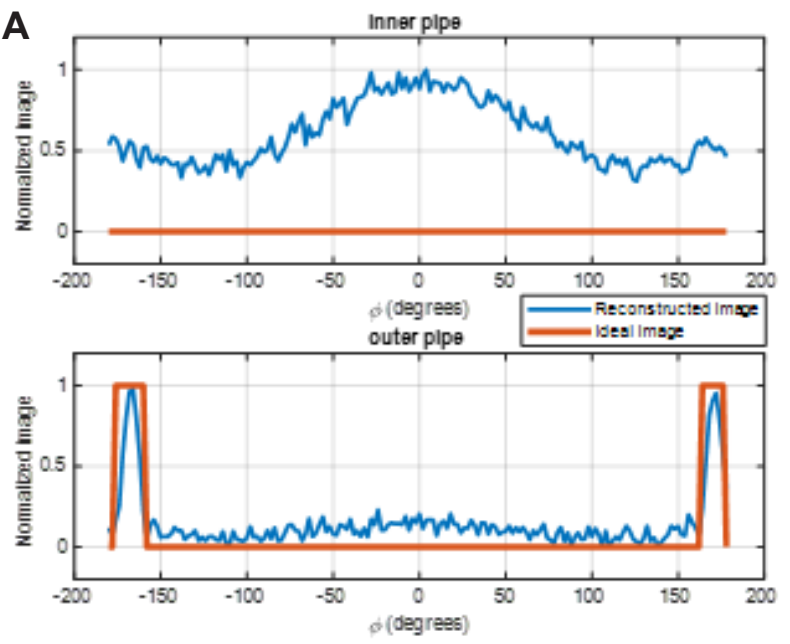

As a final step, we compute the variation of REs as the value of $E_{c c}$ increases and when the defects are at on the outer pipe or on the inner pipe. Figure 7 A shows this variation when the defects are on the outer pipe. It is observed that the value of RE increases sharply as $E_{c c}$ increases. A similar trend is observed in Figure $7 \mathrm{~B}$ when the defects are on the inner pipe.

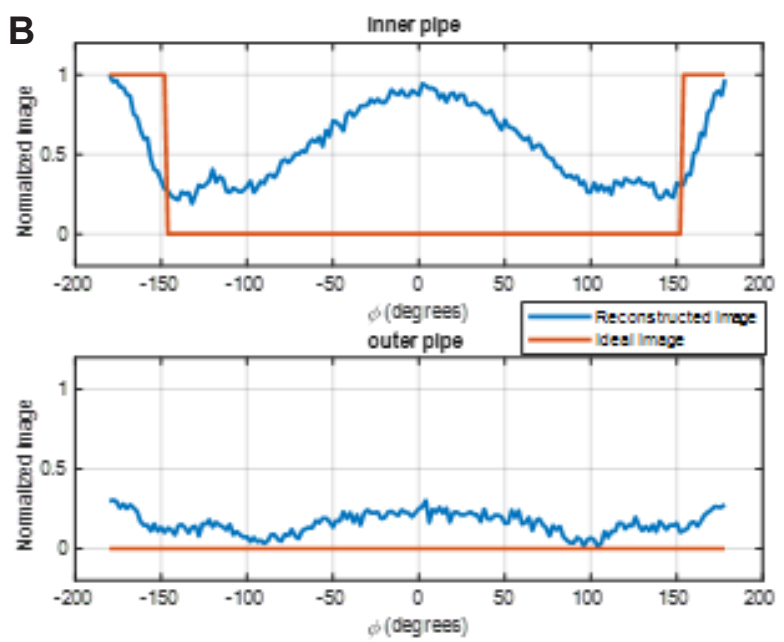

Figure 6: Reconstructed 1D images when the defects are at $\phi= \pm 170^{\circ}$ and eccentricity $=0.9 \mathrm{~mm}$ for: (A) defects on the outer pipe

(B) defects on the inner pipe.

\section{A}

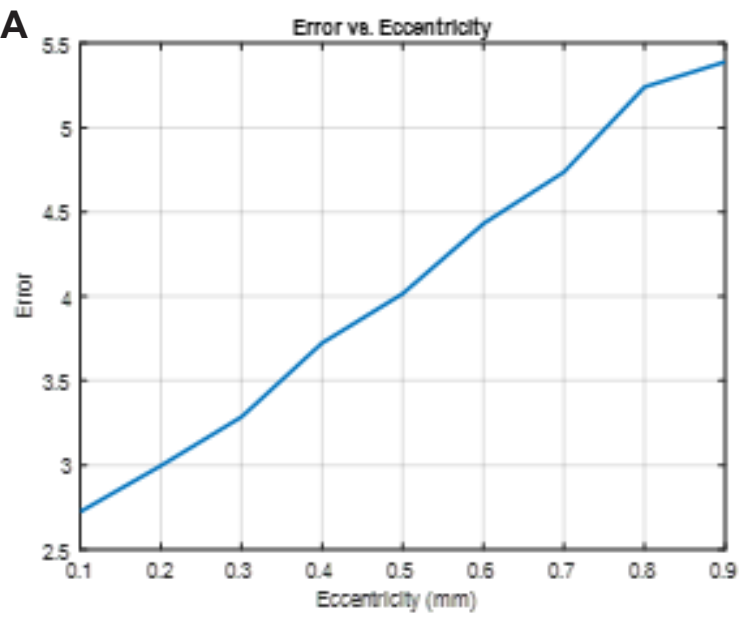

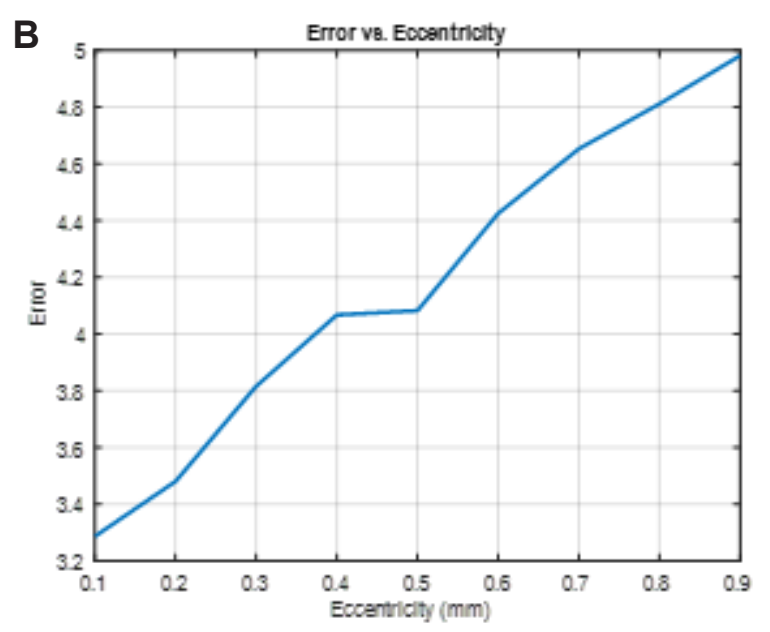

Figure 7: Variation of computed RE vs. eccentricity when defects are at $\phi= \pm 170^{\circ}$ and both defects are on
(A) outer pipe

(B) inner pipe. 


\section{DISCUSSION \& CONCLUSION}

In this paper, we studied the effect of eccentricity of the pipes on the results of the holographic microwave imaging of multiple non-metallic pipes. Microwave imaging is a non-contact method that can be used for inspection of multiple pipes and it is also safe due to the use of low-level microwave power.

In general, the results indicate that the quality of the reconstructed images is highly sensitive to non-zero eccentricity values such that even an eccentricity of $0.5 \mathrm{~mm}$ imposes image quality deteriorations. Besides, it was observed that the images of the defects on the inner pipes would be affected more seriously by non-zero eccentricity effects.

Although we employed simulated results from Altair FEKO software, we applied additive white Gaussian noise to the simulated responses to mimic real-world measurements and have a more realistic study.

Here, the study was conducted with two scenarios: (1) changing the angles of the defects while the eccentricity value is fixed and (2) changing the value of eccentricity while the positions of defects are fixed. It is worth noting that the effect of other important parameters for the considered microwave imaging setup such as thickness, radius, and permittivity of the pipes as well as angular separation of the antennas have been already studied in [15] and have been excluded here. Due to serious adverse effects of eccentricity, we plan to develop a technique to estimate the value of unknown eccentricity parameter and then reduce the effect of that on the reconstructed images. Ultimately, the goal is to develop a robust tool for NDT of non-metallic pipes that promote using these components in various industries.

\section{AUTHOR INFORMATION}

\section{Corresponding Author}

*Email: ygao21@nyit.edu

\section{Author Contributions}

Yuki Gao and Noshin Raisa have contributed in performing the parametric analysis of the eccentricity effect. Reza K. Amineh has provided the original FEKO simulation models and MATLAB codes for applying the nearfield holographic imaging. All authors have contributed in preparation of this manuscript.

\section{Funding Sources}

U.S. National Science Foundation (NSF) under Award 1920098 and the New York Institute of Technology's Institutional Support for Research and Creativity (ISRC) Grants

\section{Competing Interests}

The authors declare no competing financial and non-financial interests.

\section{ACKNOWLEDGEMENTS}

The authors thank New York Institute of Technology's Undergraduate Research and Entrepreneurship Program (UREP).

\section{ABBREVIATIONS}

CD - Calibration Defect

CFRP - Carbon Fiber Reinforced Plastic

DFT - Discrete Fourier transform

DTFT - Discrete Time Fourier Transform

FRP - Fiber Reinforced Plastic

GRE - Glass Reinforced Epoxy Resin

HDPE - High Density Polyethylene

NDT - Non-Destructive Testing

PSF - Point-Spread Function

PVC - Polyvinyl Chloride

RE - Reconstruction Error

REJ -Rubber Expansion Joint

SAR - Synthetic Aperture Radar

\section{REFERENCES}

[1] L. Tong, A. P. Mouritz, M. K. Bannister, 3D Fiber Reinforced Polymer Composites. 1st ed. (2002). 
[2] K. Murphy, D. Lowe, Evaluation of a novel microwave based NDT inspection method for polyethylene joints. in Proc. ASME Pressure Vessels Piping Conf., Baltimore, MD, U.S.A. 321-327 (2002).

[3] X. W. Zhu, J. P. Pan, L. J. Tan, Microwave scan inspection of HDPE piping thermal fusion welds for lack of fusion defects. Appl. Mech. Mater. 333-335, 1523-1528 (2013).

[4] R. Pincu, "Digital radiography and its advantages in field NDT inspections today" 17th World Conf. on Nondestructive Testing, Shanghai, China (2008).

[5] R. Zoughi, Microwave Non-Destructive Testing and Evaluation. (2000).

[6] S. Kharkovsky, R. Zoughi, Microwave and millimeter wave nondestructive testing and evaluation - Overview and recent advances. IEEE Instr. and Meas. Mag., 10(2), 26-38 (2007).

[7] D. M. Sheen, D. L. Mcmakin, T. E. Hall, Three-dimensional millimeter-wave imaging for concealed weapon detection. IEEE Trans. Microw. Theory Techn., 49(9), 1581-1592 (2001).

[8] M. T. Ghasr, K. Ying, R. Zoughi, 3D millimeter wave imaging of vertical cracks and its application for the inspection of HDPE pipes. AIP Conf. Proc., 1581(1), 1531-1536 (2014).

[9] R. K. Amineh, N. K. Nikolova, M. Ravan, Real-Time Three-Dimensional Imaging of Dielectric Bodies Using Microwave/Millimeter Wave Holography. Wiley \& IEEE Press, ISBN: 978-1-119-53886-8, (2019).

[10] R. K. Amineh, M. Ravan, H. Wu, A. Kasturi, Three-dimensional holographic imaging using data collected over cylindrical apertures. Microwave and Optical Technology Letters, 61(4), 907-911 (2019).
[11] R. K. Amineh, J. McCombe, A. Khalatpour, N. K. Nikolova, Microwave holography using point-spread functions meas-ured with calibration objects. IEEE Trans. on Inst. and Meas., 64(2), 403-417 (2015).

[12] L. E. S. Martin, A. E. Fouda, R. K. Amineh, I. Capoglu, B. Donderici, S. S. Roy, F. Hill, New high-definition frequency tool for tubing and multiple casing corrosion detection. SPE Abu Dhabi Int. Petroleum Exhibition \& Conference, (2017).

[13] Y. Renardy, D. D. Joseph, Couette flow of two fluids between concentric pipes. J. of Fluid Mechanics, 150, 381-394 (1985).

[14] R. K. Amineh, M. Ravan, R. Sharma, Nondestructive testing of nonmetallic pipes using wideband microwave measurements. IEEE Trans. Microwave Theory and Techniques, $65 w(5), 1763-1772$ (2020).

[15] H. Wu, M. Ravan, R. Sharma, J. Patel, R. $\mathrm{K}$. Amineh, Microwave holographic imaging of nonmetallic concentric pipes. IEEE Trans. Instrumentation \& Measurement, 69(10), 75947605 (2020).

[16] Altair FEKO, https://www.altair.com/feko/ 